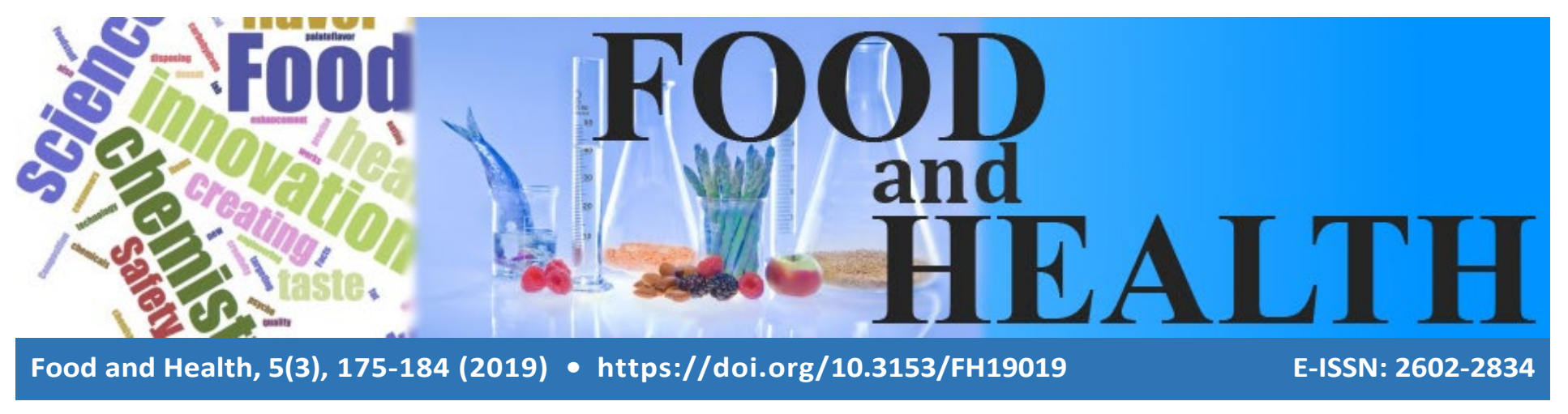

Research Article

\title{
RHEOLOGY OF FILM-FORMING SOLUTIONS AND PHYSICAL PROPERTIES OF DIFFERENTLY DEACETYLATED SALEP GLUCOMANNAN FILM
}

\begin{abstract}
Abdullah Kurt (i)
Cite this article as:

Kurt, A. (2019). Rheology of film-forming solutions and physical properties of differently deacetylated salep glucomannan film. Food and Health, 5(3), 175-184. https://doi.org/10.3153/FH19019
\end{abstract}

Bitlis Eren University, Engineering Architecture Faculty, Department of Food Engineering, 13000 Bitlis, Turkey

Submitted: 19.12 .2018

Accepted: 07.03.2019

Published online: 23.03 .2019

ORCID IDs of the authors:

A.K. 0000-0003-1452-3278

Correspondence:

Abdullah KURT

E-mail: akurt@beu.edu.tr

(๔) Copyright 2019 by ScientificWebJournals Available online at http://ifhs.scientificwebjournals.com

\begin{abstract}
The aim of this work was to evaluate the influence of deacetylation degrees (DD, 0-50-100\%) and concentrations of glycerol (G, $0-5-10 \%)$ on the film solution rheology and physical properties of salep glucomannan film. Solution rheology experiments demonstrated that the deacetylation can be used as a tool to regulate film solution flow, its spread and also coating applications. Deacetylated salep films were produced with and without glycerol. The non-plasticized film was obtained with no cracks and bubbles. The deacetylation of purified glucomannan with the aid of glycerol significantly changed the physical properties of the film by increasing its density, opacity whereas light transmittance and moisture content were decreased due to the formation of associations between acetyl free regions of the glucomannan chains. Overall, $100 \%$ DD films with $10 \%$ glycerol demonstrated better physical characteristics. Findings suggest that deacetylation is promising chemically modification method for the production of food packaging materials.
\end{abstract}

Keywords: Biodegradable film, Salep glucomannan, Deacetylation, Rheology, Physical properties 


\section{Introduction}

Deacetylation defines removing acetyl groups from glucomannan backbone by alkali treatment. Structural and functional characteristics of chemically modified glucomannan exhibits alterations such as, thermo-reversible gel formation with a higher water resistance and better mechanical properties due to the occurrence of hydrophobic interaction in addition to hydrogen bonding ( $\mathrm{Li}$ et al., 2018). This process widely performed to konjac glucomannan based biodegradable film samples, in order to overcome high water-soluble, poor mechanical properties, brittleness, and sensitivity to water, (Chen et al., 2011; Du et al., 2012; Jin et al., 2015; Li et al., 2014). As compared with other modification methods which conducted to improve film properties, deacetylation can be evaluated as a facile method without using additional polymer or hydrophobic ingredients to produce the film with better characteristics.

Salep, as another important glucomannan source, had few reports on the film properties (Ekrami \& Emam-Djomeh, 2014; Kurt and Kahyaoglu, 2014; Yilmaz and Vatansever, 2016), in addition, no research has been performed to cope with salep glucomannan film deficiencies. Salep glucomannan (SG) is produced from the tuber of the Orchidaceae family. Glucomannan is the main constituent present in the tubers. As a hydrocolloid, salep forms viscous solutions when dissolved in water due to the higher molecular weight. The structure of glucomannan consists of a linear backbone connected with $\beta-(1 \rightarrow 4)$ glycosidic bonds, which are composed of glucose and mannose units. The powder of salep also contains starch (36.31\%); protein $(4.60 \%)$; and ash (2.07\%) (Kurt and Kahyaoglu, 2015). We have conducted purification studies to isolate glucomannan and we obtained clearer and more stable solutions with better flow characteristics (Kurt and Kahyaoglu, 2017b, 2017c).

The rheological properties of the film-forming solutions is an crucial experiment because rheological parameters can affect spreadability, thickness and uniformity of liquid coating layer which applied to food products by dipping, brushing or spraying. The mechanical properties and the optimizing processing design during application were affected by flow behavior of film solutions. If the viscosity is high or gel type structure, casting of film solution in thin layers and the elimination of air bubbles will be difficult during high shear processing operations, such as pumping, filling and spraying application. It is also stated that, rheological parameters are useful for the evaluation of structure-function relationships of polysaccharide solutions systems (Chen, et al., 2009; Ma, et al., 2017a; Wu, et al., 2016). Therefore, to provide a broad understanding on the physical characteristics of edible films it is necessary to study the rheological properties of the deacetylated glucomannan film solutions due to the variations in the molecular structure.

If the film property of salep is improved by modification methods, salep will have a potential to receive much interest due to their environmentally friendly material behavior. Therefore, the novelty and significance of this study on the advance of polysaccharide based food packaging field is stated as follow:

i. The significance of deacetylation process with different degrees on the salep glucomannan film rheology and physical properties were evaluated for the first time.

ii. Moreover, the application of the different degree of deacetylation at the presence of different glycerol concentrations is also first attempt leading to reveal plasticizer effect on modified glucomannan structure.

iii. The purification process is needed so as to apply deacetylation to glucomannan. For this reason, the nine different edible films were produced from purified glucomannan. Therefore, this study also provided to observe the purification effect on the film properties of salep.

\section{Materials and Methods}

\section{Materials}

Purified glucomannan $(\mathrm{GM})\left(\mathrm{M}_{\mathrm{w}}: 1.03 \times 10^{6} \mathrm{~g} / \mathrm{mol}\right.$, PDI: 1.78) was used for differently deacetylated film production (Kurt and Kahyaoglu, 2017b, 2017c). All chemicals used in this study were of analytical grade. The ethanol, sodium hydroxide and glycerol were purchased from Sigma Chemical Co. (St. Louis, MO, USA).

\section{Film Preparation}

The solution casting method was conducted for film formation as described earlier (Kurt et al., 2017). Before deacetylation process, $1 \mathrm{~g} \mathrm{GM}(0.5 \%$, w $/ \mathrm{v})$ was dissolved in $200 \mathrm{~mL}$ distilled water, including different glycerol ratios $(0,5$ and $10 \mathrm{wt} \%$ of GM). The solution was heated up to $65^{\circ} \mathrm{C}$ and stirring of solution was maintained at this temperature for about $30 \mathrm{~min}$ to get a clear solution. Deacetylation (DD) $(0,50$ and $100 \mathrm{DD})$ was applied to the solution for each plasticizer concentration, by adding determined amount of sodium hydroxide to the solutions with the following equation (Kurt and Kahyaoglu, 2017a; Liu et al., 2010): 
$D D(\%)=\frac{W_{2} \times 43}{40 \times W_{1} \times 0.022} \times 100 \quad 1$

where $\mathrm{W}_{1}$ and $\mathrm{W}_{2}$ were the weight of $\mathrm{GM}$ and $\mathrm{NaOH}$ in $\mathrm{g}$, respectively. This equation was acquired based on the degree of acetylation of the purified glucomannan backbone which was determined to be $2.2 \%$ in this study by using the reported back-titration method (Xiao et al., 2015). The solutions $(200 \mathrm{~mL})$ at $65^{\circ} \mathrm{C}$ were then cast in petri plates $(15 \mathrm{~cm}$ diameter) and dried at $40{ }^{\circ} \mathrm{C}$ for $24 \mathrm{~h}$. The film characterization analyses were performed to all dried and peeled off films which also conditioned for at least $48 \mathrm{~h}$ at $24{ }^{\circ} \mathrm{C}$ in desiccators at $43 \%$ relative humidity $\left(\mathrm{K}_{2} \mathrm{CO}_{3}\right.$ saturated solution). The differently deacetylated (DD; 0,50 and 100\%) GM film samples with different glycerol (G: 0, 5 and 10\%) concentration were coded as 0DD-0G, 0DD-5G, 0DD-10G, 50DD-0G, 50DD-5G, 50DD-10G, 100DD-0G, 100DD-5G 100DD-10G, respectively.

\section{Rheological Properties of Film Solutions}

The rheological properties of film solutions were determined by using a rheometer (HAAKE Mars III; Thermo Scientific, Germany) that was equipped with a Peltier heating system with a cone and plate configuration. Samples were sheared continuously at a rate ranging from 0 to $100 \mathrm{~s}^{-1}$ for $2 \mathrm{~min}$ at $65^{\circ} \mathrm{C}$ to fit the data to the Ostwald-de Waele model: $\tau=K . \dot{\gamma}^{n} \quad \mathbf{2}$

where $\tau$ is the shear stress $(\mathrm{Pa}), \dot{\gamma}$ is the shear rate $\left(\mathrm{s}^{-1}\right), K$ is the consistency coefficient $\left(\mathrm{Pa} \cdot \mathrm{s}^{\mathrm{n}}\right)$, and $n$ is the flow behaviour index (dimensionless).

\section{Film Thickness}

Film thickness was measured by a digital micrometer (Mitutoyo, Manufacturing Co. Ltd., Japan) at ten random locations of each film.

\section{Film Density}

The film density was determined from the ratio between the weight and volume (thickness $\times$ area). The density experiments were conducted in triplicate.

\section{Film Moisture Content}

To moisture content determination, the percentage of water removed from the initial mass sample was analyzed gravimetrically by drying the samples at $105^{\circ} \mathrm{C}$ for $24 \mathrm{~h}$ (Kurt and Kahyaoglu, 2014).

\section{Film Color}

The color of the films was determined using a colorimeter (Colorflex, EZ, USA) in terms of lightness $\left(L^{*}\right)$, redness/greenness $\left(a^{*}\right)$, and yellowness/blueness $\left(b^{*}\right)$ values.
The total color difference $\left(\Delta E^{*}\right)$ was calculated using the following equation:

$\Delta E^{*}=\sqrt{\left(L^{*}-L\right)^{2}+\left(a^{*}-a\right)^{2}+\left(b^{*}-b\right)^{2}}$

where $L^{*}, a^{*}$, and $b^{*}$ are the standard color parameter values (93.61, -0.78 and 2.23, respectively) and $L, a$, and $b$ are the color parameter values of the sample. The measurements were repeated six times for each film.

\section{Light Transmittance and Opacity of Film}

The barrier properties of films against visible light were measured at wavelengths ranging between 300 and $800 \mathrm{~nm}$, using Cary 60 UV-visible spectrophotometer (Agilent Technologies, Victoria, Australia). The samples were cut into rectangular pieces based on the lateral area of the spectrophotometer test cell and placed in the test cell. The reference value was determined from an empty test cell. The opacity value of the films was calculated by dividing the absorbance at $600 \mathrm{~nm}$ by the film thickness (mm) (Kurt and Kahyaoglu, 2014). All determinations were performed in triplicate.

\section{Results and Discussion}

\section{Rheological Behavior of Film Solutions}

The acceptable flow characteristic of the film-forming solution is being a moderate viscosity because high or low viscosity will lead to non-uniform film production (Wu, et al., 2013). Some authors have suggested a viscosity lower than $700 \mathrm{mPas}$ for coating applications (Nair et al., 2011) and the appropriate viscosity is $1000-10,000 \mathrm{mPa}$ s for other processing conditions of film solutions such as mixing, pumping and transfer to casting line and spreading the solution smoothly (Rossman, 2009). The apparent viscosity (AV) and parameters of Ostwald-de Waele model such as consistency coefficient (CC) and flow behavior index (FBI) of 9 different film solutions were presented in Fig.1. The parameters were found higher than the film solution produced by unpurified salep even at one third of concentration (Kurt and Kahyaoglu, 2014) as a results of interaction between glucomannan chains by means of removing impurities (Kurt and Kahyaoglu, 2015) which indicated the possibilities of lower usage of salep to obtained desired viscosity (Kurt and Kahyaoglu, 2017b).

Increasing glycerol concentration affected differently $\mathrm{AV}$, $\mathrm{CC}$ and FBI values of deacetylated samples (Figure 1A). The addition of $5 \%$ glycerol decreased the values of AV and $\mathrm{CC}$ of samples. However, the decrease in these parameters proceeded for only 100DD samples at further glycerol addition $(10 \%)$. The $n$ values ranged between $0.70-0.77$, indicating pseudoplastic behavior of samples (Figure 1A) 
(Saricaoglu et al., 2018) which associated with the destruction effect of shearing on polysaccharide macromolecules composed of a large number of hydroxyl groups in the network (Ma et al., 2017b). Only 100DD-10G ( $\mathrm{n}=0.77$ ) sample had lower pseudoplastic flow behavior, AV and CC values. Therefore, this solution flows and spreads more easily, reflecting that the intermolecular interaction between glucomannan polysaccharides is destroyed by glycerol. The inhibition effect of glycerol was more apparent for 100DD. At lower DD, glycerol increment may be provided forming new hydrogen bonds and preserved their characteristics.

Increasing DD at the same glycerol content caused to same decrement trend for the AV parameters (Figure 1B). The decrease in $\mathrm{AV}$ and $\mathrm{CC}$ with removing acetyl groups were reported for salep and konjac GM solutions and the results were associated with the decrease of the entanglement force between GM molecules (Kurt and Kahyaoglu, 2017a; Wang et al., 2014). Higher hydrogen bonds between GM and water occurred as results of higher acetyl contents which cause a higher excluded-volume force reducing hydrogen bonds between GM chains. When acetyl group contents changed to a lower degree by deacetylation, the volume of the polymeric network can be diminished (Kurt and Kahyaoglu, 2017a). The decrease in $\mathrm{CC}$ values with deacetylation from 0 to 50DD was more apparent at all glycerol concentration and the further increase in DD had no significant effect on the 0 and $5 \%$ glycerol included samples but proceeding in the decrement of CC was observed at $10 \%$ glycerol concentration. After deacetylation, the presence of glycerol had an additive effect on these decrements because in glycerol added polysaccharide system, there is competition for hydrogen bonds between polysaccharide chains and polysaccharideplasticizer; and direct interaction between polysaccharide chains are partly reduced, resulting in lower viscosity (Peressini et al., 2003). As a result, pronounced lowering effect of deacetylation on consistency and pseudoplastic behavior could be stated for the higher glycerol ratios. Additionally, the revealing of decreasing effect of deacetylation process on film solution viscosity may eliminate the difficulties (high viscosity) of preparation of film solutions at higher glucomannan concentration, provided lower concentration dependency.

\section{Overall Evaluation of Film Appearance}

The production of glucomannan (GM) films with deacetylation was achieved without and with 5 and $10 \%$ glycerol additions. The films had visually continuous and homogeneous with no cracks, bubbles, or visible phase separation and they were easily removed from the plates (Figure 2). The easily peeled off non-plasticized films were not brittle and hard indicated that GM was a good matrix-forming material. The possibilities of cohesive film formation of KGM without plasticizer but failure for galactomannan (locust bean and guar gum) were stated by Mikkonen (2009). The other critical point was that fully deacetylation of glucomannan (100DD) provided gel formation of film solutions as result of the aggregation of GM molecules (Kurt and Kahyaoglu, 2017a).

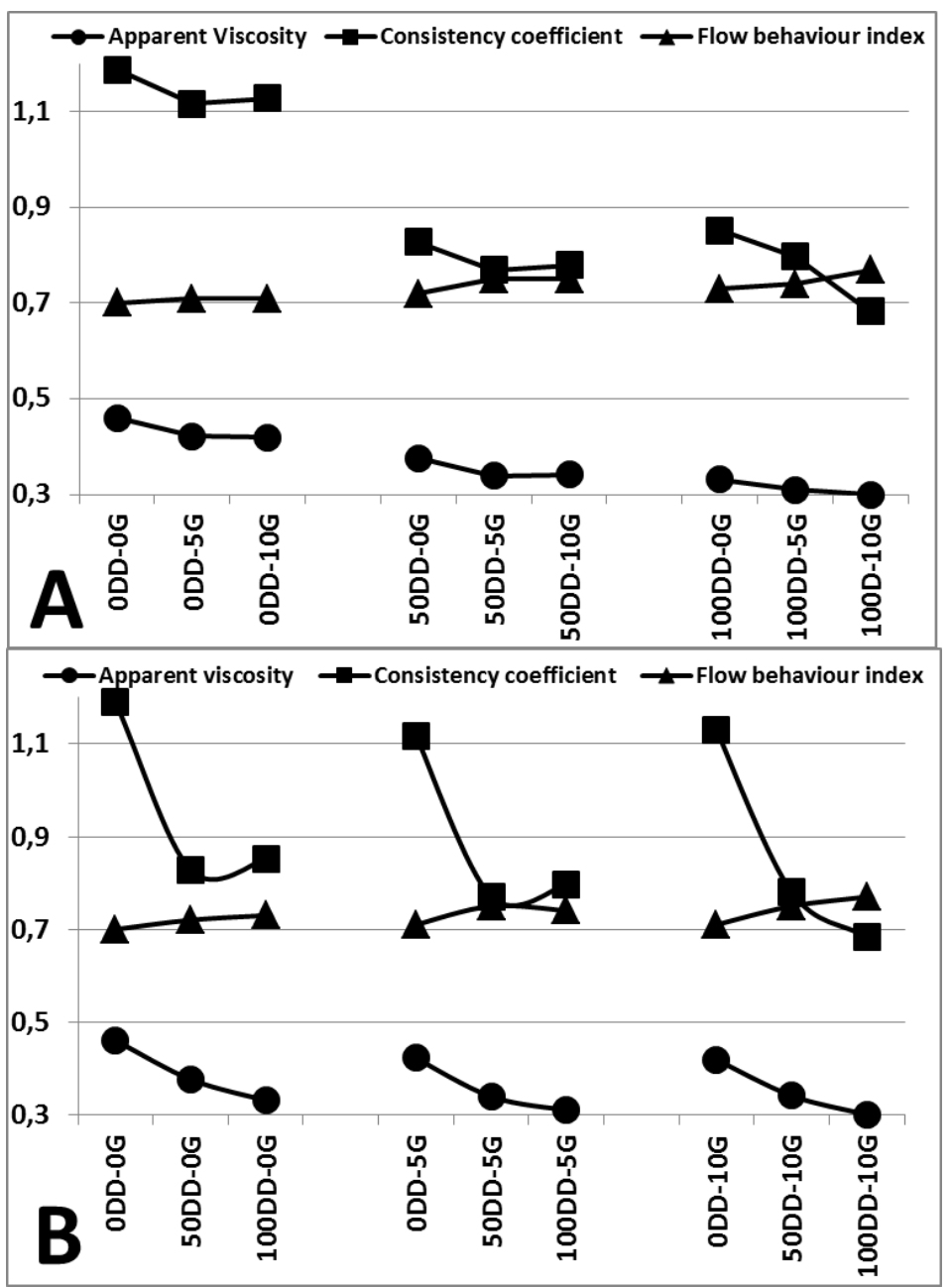

Figure 1. Apparent viscosity $\left(30 \mathrm{~s}^{-1}\right.$, Pas), consistency coefficient $\left(K, \mathrm{~Pa} . \mathrm{s}^{\mathrm{n}}\right)$ and flow behaviour index $(n)$ of film solutions (A) at same deacetylation degree (DD, \%) and (B) at the same glycerol concentration $(\mathrm{G}, \%)$ 


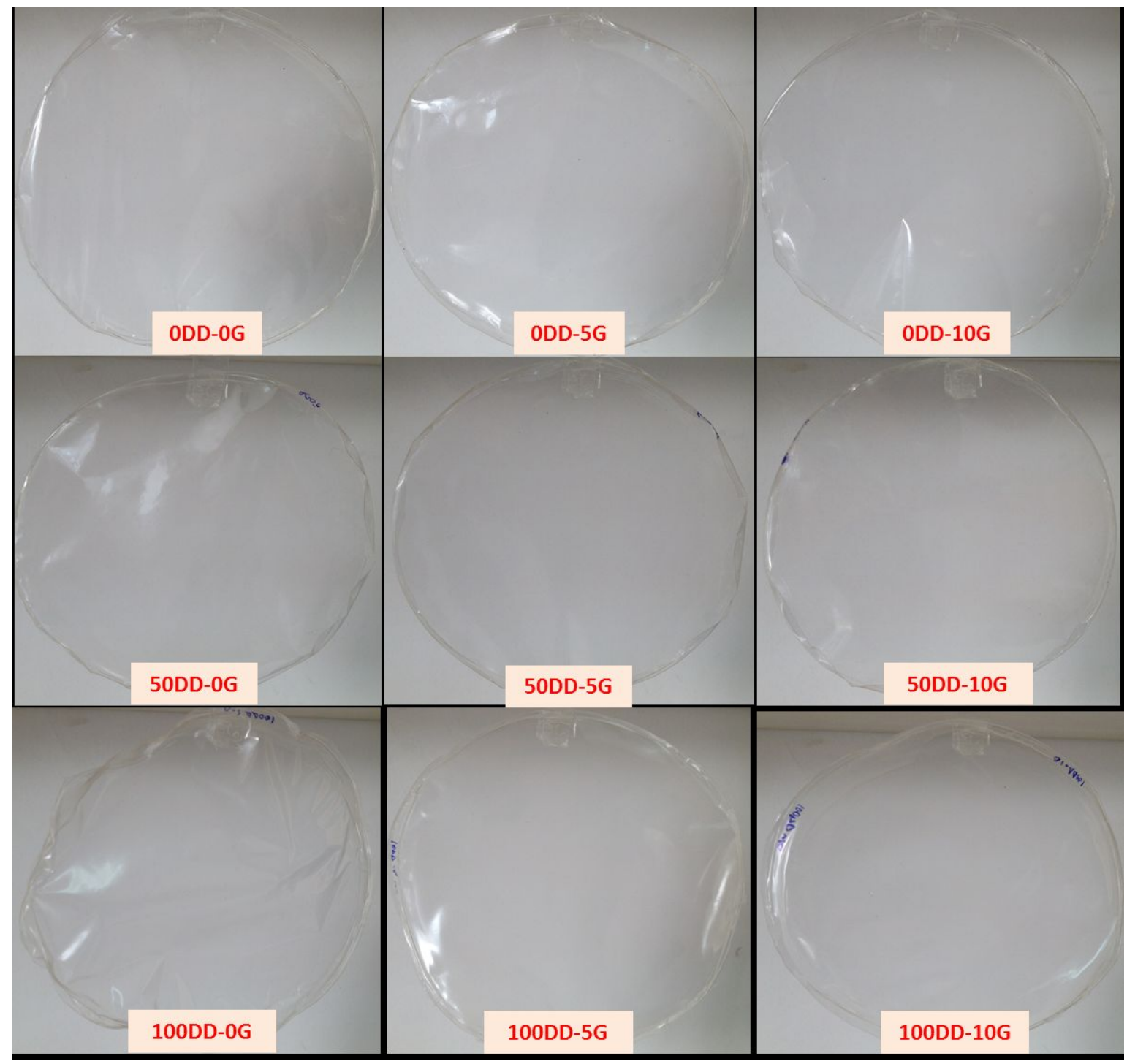

Figure 2. Physical appearance of salep glucomannan film samples as a function of deacetylation degree (DD, \%) and glycerol content $(\mathrm{G}, \%)$

\section{Film Thickness}

The thickness values of the films varied between 33.85 and $50.66 \mu \mathrm{m}$ (Figure 3). Thickness values of the unmodified films increased with the glycerol concentration but decreasing trend was observed for the deacetylated samples. According to the literature, increasing film thickness with the glycerol concentration was generally attributed to the more adsorbed water due to its hydrophilic character. This could be observed at very high glycerol content. However, in this study the water content of unmodified films decreased with glycerol. Therefore, increasing film thickness could be related to the extending the structure of film through increasing molecular volume of network due to the increasing the presence of glycerol content between the macromolecular chains (Mikkonen, 2009; Thakhiew et al., 2010), further increase in glycerol $(10 \%<)$ may be responsible for the higher water content and free volume of a system (Lai et al., 2006).

The lower viscosity values of deacetylated film solutions suggest thinner film production (Piermaria et al., 2009). In general, deacetylated samples had lower thickness values for the same glycerol contents as compared with control samples, possibly formation of associations between acetyl free regions of the GM backbone (Liu et al., 2010), thus restructuring the polymer organization to a less expanded structure with decreased volume, resulting in thinner polymer films (Razavi et al., 2015). The effect of removing ace- 
tyl groups from the GM backbone on thickness was significant at higher glycerol content. The lowest thickness values observed for the fully deacetylated samples with the presence of $10 \%$ glycerol (gelled film solution). Deacetylation of GM allows acetyl-free regions of the backbone to associate, leading to the formation of junction zones and a threedimensional network (Huang et al., 2015). Glycerol addition had improving effect on the mentioned formation by more hydrogen bonding during film forming process, providing further rigidity for the deacetylated film.

\section{Film Density}

The thinner films showed higher density values, due to the lower volume (Razavi et al., 2015). However, increased density of unmodified films (higher film volume) with glycerol increment could be attributed to the lower water content of that sample. Despite using same polymer concentration with no variations in molecular weight, higher density values of deacetylated films could be explained with increased interaction between the molecular chains of the glucomannan, resulted in different polymeric film structure production (Pelissari et al., 2013). This result is consistent with the water barrier properties of films due to the reduced interstitial spacing within the matrix of polymeric films and, consequently, producing lower water transmission. The higher density value $\left(2.05 \mathrm{~g} / \mathrm{cm}^{3}\right)$ than previously reported unpurified salep film density $\left(1.27 \mathrm{~g} / \mathrm{cm}^{3}\right)$ for the same glycerol content (10\%) (Kurt and Kahyaoglu, 2014) indicated improving the effect of removing impurities on GM interactions.

\section{Film Moisture Content}

It is evident that the presence of glycerol and modification of GM backbone with alkali affected both the interaction of polymer matrix and its hydrophilic nature. Water contents of films shown in Fig. 3 decreased with increasing glycerol contents which could be attributed to the diminishing ability of the GM granules to absorb water due to the small molecular size of glycerol allowing more hydrogen bonding instead of allowing higher free volume for more water absorption at higher glycerol concentration than in this study (Lai et al., 2006). On the other hand, deacetylation slightly lowered water values of non-plasticized film while at the presence of glycerol removing acetyl groups in $50 \%$ ratio had no effect on this value. The ability of the lowest water susceptibility occurred when acetyl groups were completely removed from film included $10 \%$ glycerol. This modification with the presence of glycerol probably altered GM conformation, making the polymer network lower hydrophilic as was observed with the water absorption, solubility and improved water barrier properties in this study. The lower moisture content $(9.0 \%)$ of $10 \%$ glycerol included purified salep films than unpurified salep (19.13\%) (Kurt and Kahyaoglu, 2014) could be explained with the decreasing hydrophilic character of salep by removing impurities (such as starch, protein and ash) and also hindering the formation of porous structure, which weakens water retention by capillarity (Pelissari et al., 2013). Moisture content decrement was also attributed to the diminishing the hydrophilic groups as a result of increased interaction between constituents of film mixture which in turn decreased the interaction between polymeric materials and water molecules (Liu et al., 2017).

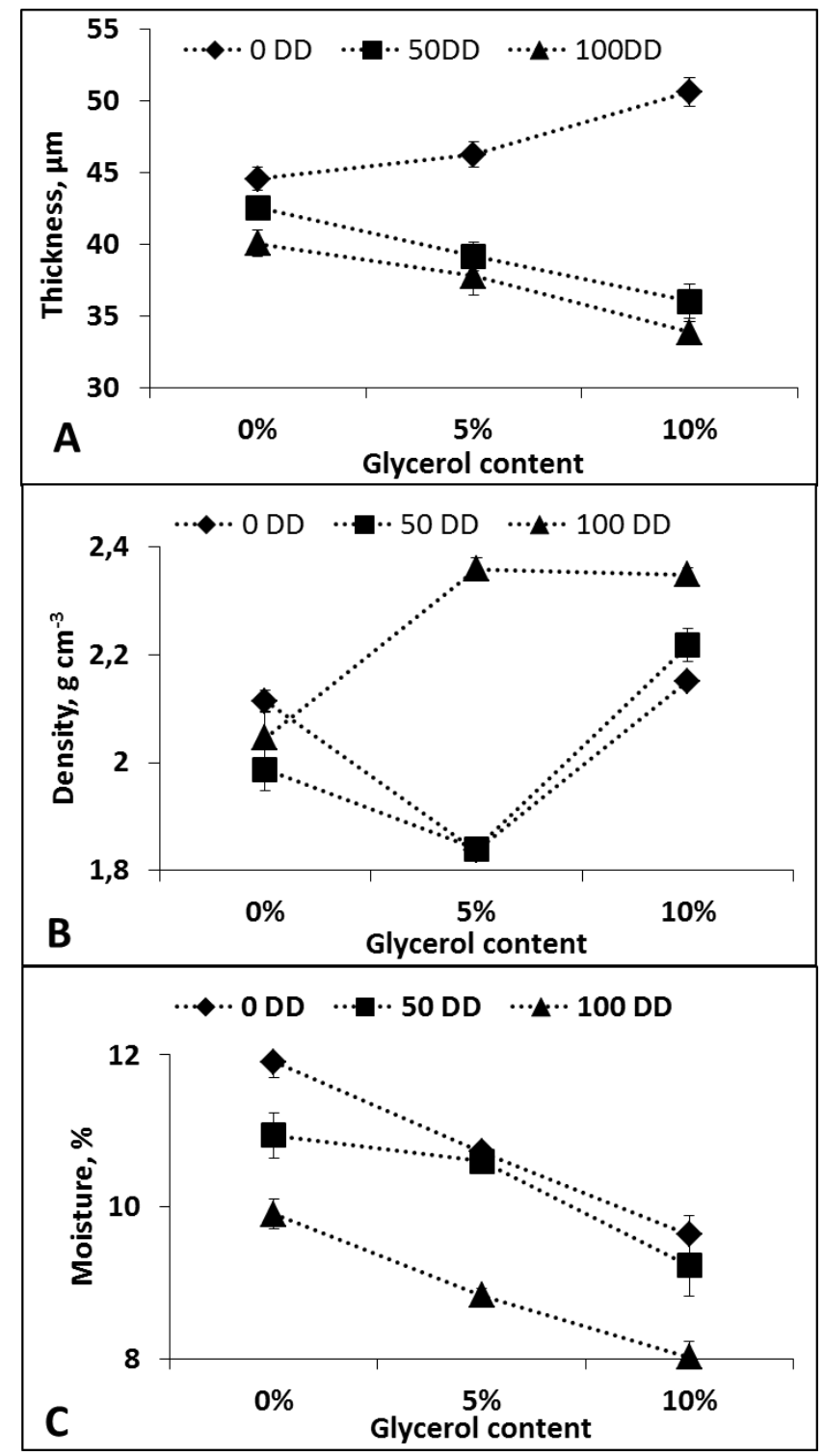

Figure 3. (A) Thickness, (B) density, and (C) moisture content of salep glucomannan film samples as a function of deacetylation degree (DD, \%) and glycerol content $(\mathrm{G}, \%)$ 


\section{Film Color}

Optical properties such as color are crucial especially in the production of food packaging for the consumer preference and also crucial for the storage of food up to its sensitive character to the light. Visually, all films were almost clear and transparent and no significant differences in the color parameters and total color differences were determined. The mean values of parameters of 9 different films as follows: $L^{*}=90.62 \pm 0.14, a^{*}=-0.91 \pm 0.02, b^{*}=3.52 \pm 0.23$, and $\Delta E^{*}=3.26 \pm 0.20$. The high lightness $L^{*}$ value indicates the high transparency of samples, which is a desirable property for the edible film packaging and coatings. The negative $a^{*}$ and close to zero value states the absence of characteristic tones of red color and positive $b^{*}$ value shows yellow color. Consistent results with the our study were reported by Razavi et al. (2015) for sage seed gum film as plasticizer independency of color parameters. The degree of total color difference from the standard color plate $\left(\Delta E^{*}\right)$ indicates the high clearness of film at low degree. Increased clearness with glycerol addition but lower clearness values than our results were determined for the exopolysaccharide films of kefiran $\left(\Delta E^{*}=9-15\right)$ (Ghasemlou et al., 2011). The independence of film color parameters to deacetylation and glycerol degrees may be probably associated with low water contents of modified films, which had no changing effect on the reflection of light at the film surface in spite of structural changes.

\section{Light Transmittance of Film}

The differences in the deacetylation degree and glycerol concentration affected visible light transmission of samples (Figure 4A) because the arrangement or alignments of polymer in film network most likely have a role on this property. An increasing content of glycerol lowered the visible light transmission of 0DD samples. The similar phenomenon was stated by Liang and Wang (2017). However, glycerol increment resulted in opposite results for the deacetylated samples such as (i) raising effect on 50DD films and (ii) no effect on light transmission of fully deacetylated film (Figure 4A). For the non-plasticized film conditions, deacetylated samples exhibited lower light transmission but similar values observed for 50DD and 100DD. Deacetylated samples revealed different behaviors after glycerol addition. As compared with 0DD, 50\% deacetylated samples had high transmission but 100DD showed lower light transmission for the plasticized samples. (Figure 4A). The differences between deacetylated samples were clearer at $10 \%$ glycerol concentration. Film 50DD-10G exhibited the highest visible light transmission, while films 100DD-5G and 100DD-10G showed the lowest visible light transmission in the high wavelength. The enhanced interaction between GM molecules in the film matrix by deacetylation and the presence of glycerol at this range caused to more absorption of the energy of incoming light, thus impeding the visible light transmission through the films was determined (Soo and Sarbon, 2018). Similar with this study, deacetylation with a degree of $52 \%$ increased the transparency of konjac glucomannan (KGM) film but increasing deacetylation degree (70 and $86 \%$ ) decreased film transparency (Jin et al., 2015).

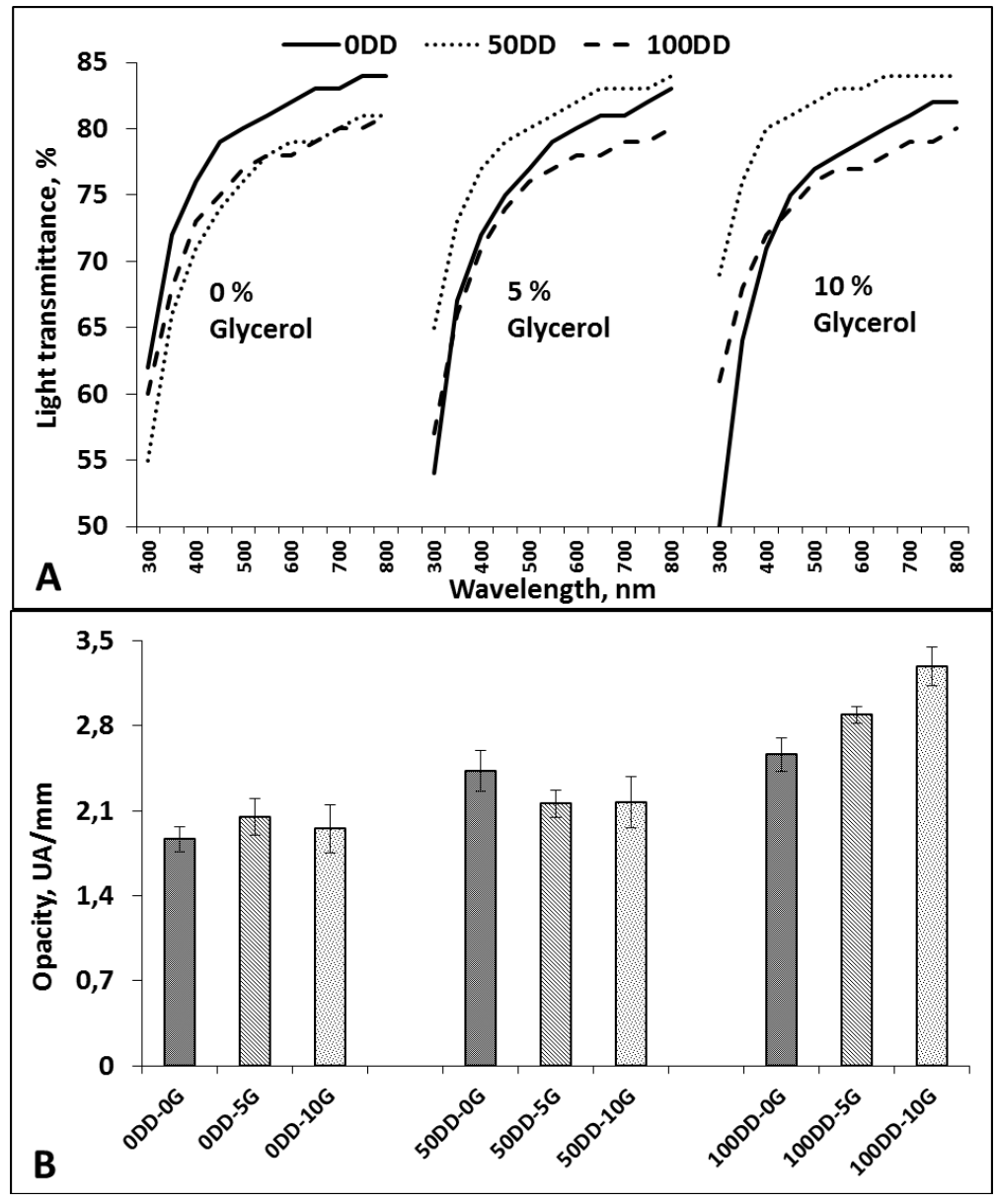

Figure 4. (a) Light transmittance, and (b) opacity of salep glucomannan film samples as a function of deacetylation degree (DD, \%) and glycerol content $(\mathrm{G}, \%)$

\section{Film Opacity}

Opacity values of all film formulation are presented in Figure 4B. The results agreed with the light transmittance of films. Opaqueness increment by deacetylation was observed at the presence of $10 \%$ glycerol more specifically. The similar increase in the opacity was also reported for the films incorporated with polyphenols which improve absorption ability by means of their aromatic groups (Liu et al., 2017) and also the addition of different polymeric materials which 
resulted in light scattering (Condés et al., 2018). However, the results in this study were attributed to the enhanced interaction between GM molecules by deacetylation which prevented the light to transmit through the films consequently film opacity increased. This suggested phenomenon between GM molecules reduced the biopolymer-water interactions, providing the formation of an opaque polymer matrix, consistent with the lower water contents of deacetylated films (Pelissari et al., 2013). Additionally, the increase in film opacity by glycerol incorporation was significant for the fully deacetylated glucomannan based film which revealed the formation of more tightly packed polymer network (Chang and Nickerson, 2015). Lower light transmittance and high opacity values of deacetylated salep films made it preferable as compared with unmodified salep for the protection of packaged food against light catalyzed degradation reactions formations. Regarding the purification effect on film properties, it could be stated that unpurified film had higher opacity values (3.74 UA/mm) (Kurt and Kahyaoglu, 2014) than purified salep (1.95 UA/mm) obtained in this study at similar glycerol content $(10 \%)$, due to the presence of impurities such as starch and protein (Mikkonen et al., 2010).

\section{Conclusion}

For the first time, salep glucomannan films were successfully synthesized with various degrees of deacetylation and glycerol concentrations. The non-plasticized film was produced from purified salep with no cracks, bubbles, or visible phase separation. Rheological experiments showed that deacetylation process decreased consistency and pseudoplastic behavior of film solutions, resulting in lower concentration dependency during film production. Acetyl free region of the glucomannan backbone leads to more associations so the thinner films with high density were obtained with deacetylation. Deacetylation had no effect on color parameters but decreased light transmittance and increased the opacity of films. By controlling the degree of acetylation and glycerol ratio, film solution rheology and physical properties of films can be regulated by tailoring biopolymers to give specific film properties. Solubility, barrier, mechanical and structural characteristics will be evaluated in further study.

\section{Compliance with Ethical Standard}

Conflict of interests: The author declared no potential conflicts of interest with respect to the research, authorship, and/or publication of this article.

Financial disclosure: The author received no financial support for the research, authorship, and/or publication of this article.

\section{References}

Chang, C., Nickerson, M. T. (2015). Effect of protein and glycerol concentration on the mechanical, optical, and water vapor barrier properties of canola protein isolatebased edible films. Food Science and Technology International, 21(1), 33-44.

Chen, C.-H., Kuo, W.-S., Lai, L.-S. (2009). Rheological and physical characterization of film-forming solutions and edible films from tapioca starch/decolorized hsiantsao leaf gum. Food Hydrocolloids, 23(8), 2132-2140.

Chen, J., Li, J., Li, B. (2011). Identification of molecular driving forces involved in the gelation of konjac glucomannan: Effect of degree of deacetylation on hydrophobic association. Carbohydrate Polymers, 86(2), 865-871.

Condés, M. C., Añón, M. C., Dufresne, A., Mauri, A. N. (2018). Composite and nanocomposite films based on amaranth biopolymers. Food Hydrocolloids, 74, 159167.

Du, X., Li, J., Chen, J., Li, B. (2012). Effect of degree of deacetylation on physicochemical and gelation properties of konjac glucomannan. Food Research International, 46(1), 270-278.

Ekrami, M., Emam-Djomeh, Z. (2014). Water vapor permeability, optical and mechanical properties of salep-based edible film. Journal of Food Processing and Preservation, 38(4), 1812-1820.

Ghasemlou, M., Khodaiyan, F., Oromiehie, A., Yarmand, M. S. (2011). Development and characterisation of a new biodegradable edible film made from kefiran, an exopolysaccharide obtained from kefir grains. Food Chemistry, 127(4), 1496-1502.

Huang, Y.-C., Chu, H.-W., Huang, C.-C., Wu, W.-C., Tsai, J.-S. (2015). Alkali-treated konjac glucomannan film as a novel wound dressing. Carbohydrate Polymers, 117(Supplement C), 778-787.

Jin, W., Song, R., Xu, W., Wang, Y., Li, J., Shah, B. R., Li, Y., Li, B. (2015). Analysis of deacetylated konjac glucomannan and xanthan gum phase separation by film forming. Food Hydrocolloids, 48, 320-326. 
Kurt, A., Kahyaoglu, T. (2014). Characterization of a new biodegradable edible film made from salep glucomannan. Carbohydrate Polymers, 104(Supplement C), 50-58.

Kurt, A., Kahyaoglu, T. (2015). Rheological properties and structural characterization of salep improved by ethanol treatment. Carbohydrate Polymers, 133(Supplement C), 654-661.

Kurt, A., Kahyaoglu, T. (2017a). Gelation and structural characteristics of deacetylated salep glucomannan. Food Hydrocolloids, 69(Supplement C), 255-263.

Kurt, A., Kahyaoglu, T. (2017b). Purification of glucomannan from salep: Part 1. Detailed rheological characteristics. Carbohydrate Polymers, 168(Supplement C), 138-146.

Kurt, A., Kahyaoglu, T. (2017c). Purification of glucomannan from salep: Part 2. Structural characterization. Carbohydrate Polymers, 169, 406416.

Kurt, A., Toker, O. S., Tornuk, F. (2017). Effect of xanthan and locust bean gum synergistic interaction on characteristics of biodegradable edible film. International Journal of Biological Macromolecules, 102(Supplement C), 1035-1044.

Lai, H. C., Karim, A. A., Chee, C. S. (2006). Effects of water-glycerol and water-sorbitol interactions on the physical properties of konjac glucomannan films. Journal of Food Science, 71(2), E62-E67.

Li, J., Ma, J., Chen, S., He, J., Huang, Y. (2018). Characterization of calcium alginate/ deacetylated konjac glucomannan blend films prepared by $\mathrm{Ca}^{2+}$ crosslinking and deacetylation. Food Hydrocolloids, 82, 363-369.

Li, J., Ye, T., Wu, X., Chen, J., Wang, S., Lin, L., Li, B. (2014). Preparation and characterization of heterogeneous deacetylated konjac glucomannan. Food Hydrocolloids, 40, 9-15.

Liang, T., Wang, L. (2017). Preparation and characterization of a novel edible film based on Artemisia sphaerocephala Krasch. gum: Effects of type and concentration of plasticizers. Food Hydrocolloids, 77, 501-508.
Liu, F., Luo, X., Lin, X. (2010). Adsorption of tannin from aqueous solution by deacetylated konjac glucomannan. Journal of Hazardous Materials, 178(1), 844-850.

Liu, J., Li, B., Zhu, B., Fu, R. H., Yuan, L. N., Huang, W., Ma, M. H. (2010). Study on properties and aggregation structures of deacetylated konjac glucomannan / chitosan hydrochloride absorbent blend gel films. Journal of Applied Polymer Science, 115(3), 15031509 .

Liu, J., Liu, S., Wu, Q., Gu, Y., Kan, J., Jin, C. (2017). Effect of protocatechuic acid incorporation on the physical, mechanical, structural and antioxidant properties of chitosan film. Food Hydrocolloids, 73, 90-100.

Ma, Q., Du, L., Yang, Y., Wang, L. (2017a). Rheology of film-forming solutions and physical properties of tara gum film reinforced with polyvinyl alcohol (PVA). Food Hydrocolloids, 63, 677-684.

Ma, Q., Du, L., Yang, Y., Wang, L. (2017b). Rheology of film-forming solutions and physical properties of tara gum film reinforced with polyvinyl alcohol (PVA). Food Hydrocolloids, 63(Supplement C), 677-684.

Mikkonen, K. S. (2009). Mannans as film formers and emulsion stabilizers University of Helsinki, Helsinki University Printing House.

Mikkonen, K. S., Heikkilä, M. I., Helén, H., Hyvönen, L., Tenkanen, M. (2010). Spruce galactoglucomannan films show promising barrier properties. Carbohydrate Polymers, 79(4), 1107-1112.

Nair, S. B., Jyothi, A. N., Sajeev, M. S., Misra, R. (2011). Rheological, mechanical and moisture sorption characteristics of cassava starch-konjac glucomannan blend films. Starch/Staerke, 63(11), 728-739.

Pelissari, F. M., Andrade-Mahecha, M. M., Sobral, P. J. d. A., Menegalli, F. C. (2013). Comparative study on the properties of flour and starch films of plantain bananas (Musa paradisiaca). Food Hydrocolloids, 30(2), 681690.

Peressini, D., Bravin, B., Lapasin, R., Rizzotti, C., Sensidoni, A. (2003). Starch-methylcellulose based edible films: rheological properties of film-forming dispersions. Journal of Food Engineering, 59(1), 2532 . 
Piermaria, J. A., Pinotti, A., Garcia, M. A., Abraham, A. G. (2009). Films based on kefiran, an exopolysaccharide obtained from kefir grain: Development and characterization. Food Hydrocolloids, 23(3), 684-690.

Razavi, S. M. A., Mohammad Amini, A., Zahedi, Y. (2015). Characterisation of a new biodegradable edible film based on sage seed gum: Influence of plasticiser type and concentration. Food Hydrocolloids, 43(Supplement C), 290-298.

Rossman, J. M. (2009). Commercial Manufacture of Edible Films. In K. C. Huber, M. E. Embuscado (Eds.), Edible Films and Coatings for Food Applications (pp. 367390). New York, NY: Springer New York.

Saricaoglu, F. T., Tural, S., Gul, O., Turhan, S. (2018). High pressure homogenization of mechanically deboned chicken meat protein suspensions to improve mechanical and barrier properties of edible films. Food Hydrocolloids, 84, 135-145.

Soo, P. Y., Sarbon, N. M. (2018). Preparation and characterization of edible chicken skin gelatin film incorporated with rice flour. Food Packaging and Shelf Life, 15, 1-8.

Thakhiew, W., Devahastin, S., Soponronnarit, S. (2010). Effects of drying methods and plasticizer concentration on some physical and mechanical properties of edible chitosan films. Journal of Food Engineering, 99(2), 216-224.
Wang, S., Zhan, Y., Wu, X., Ye, T., Li, Y., Wang, L., Chen, Y., Li, B. (2014). Dissolution and rheological behavior of deacetylated konjac glucomannan in urea aqueous solution. Carbohydrate Polymers, 101(Supplement C), 499-504.

Wu, C., Tian, J., Li, S., Wu, T., Hu, Y., Chen, S., Sugawara, T., Ye, X. (2016). Structural properties of films and rheology of film-forming solutions of chitosan gallate for food packaging. Carbohydrate Polymers, 146, 1019.

Wu, J., Zhong, F., Li, Y., Shoemaker, C. F., Xia, W. (2013). Preparation and characterization of pullulan-chitosan and pullulan-carboxymethyl chitosan blended films. Food Hydrocolloids, 30(1), 82-91.

Xiao, M., Dai, S., Wang, L., Ni, X., Yan, W., Fang, Y., Corke, H., Jiang, F. (2015). Carboxymethyl modification of konjac glucomannan affects water binding properties. Carbohydrate Polymers, 130(Supplement C), 1-8.

Yilmaz, M. T., Vatansever, C. (2016). Three interval thixotropy test to determine structural regeneration of a glucomannan based hydrocolloid film at air/water interface: Interfacial, molecular, thermal and surface characterization. Food Hydrocolloids, 61, 458-468. 